\title{
Polyethylene Glycols as Efficient Media for Decarboxylative Nitration of $\alpha, \beta$-Unsaturated Aromatic Carboxylic Acids by Ceric Ammonium Nitrate in Acetonitrile Medium: A Kinetic and Mechanistic Study
}

\author{
K. Ramesh, S. Shylaja, K. C. Rajanna, P. Giridhar Reddy, and P. K. Saiprakash \\ Department of Chemistry, Osmania University, Hyderabad, Andhra Pradesh 500 007, India
}

Correspondence should be addressed to K. C. Rajanna; kcrajannaou@yahoo.com

Received 19 September 2012; Accepted 5 December 2012

Academic Editor: Leonardo Palmisano

Copyright (C) $2013 \mathrm{~K}$. Ramesh et al. This is an open access article distributed under the Creative Commons Attribution License, which permits unrestricted use, distribution, and reproduction in any medium, provided the original work is properly cited.

Polyethylene glycols (PEGs) were found to be efficient media for decarboxylative nitration of $\alpha, \beta$-unsaturated aromatic carboxylic acids by ceric ammonium nitrate (CAN) in acetonitrile to give $\beta$-nitrostyrene derivatives. Kinetics of the reaction exhibited second order kinetics with a first order dependence on [CAN] and [substrate]. Reactions were too sluggish to be studied in the absence of PEG; therefore detailed kinetics were not taken up. Reaction times were reduced from $24 \mathrm{hrs}$ to few hours. The catalytic activity was found to be in the increasing order PEG-300 > PEG-400 > PEG-600 > PEG-200. Mechanism of PEG-mediated reactions was explained by Menger-Portnoy's scheme as applied in micellar kinetics.

\section{Introduction}

Cerium (IV) ammonium nitrate (CAN) is one of the most important reagents used for organic synthesis [1-4]. The formula of cerium (IV) ammonium nitrate, $\left[\left(\mathrm{NH}_{4}\right)_{2}\left[\mathrm{Ce}\left(\mathrm{NO}_{3}\right)_{6}\right]\right.$, reflects that the cerium (IV) ion is surrounded by six nitrate groups and the ammonium ions are counterions to compensate for the negative charge of the hexanitratocerate (IV) coordinating unit. CAN is a one-electron oxidation reagent. Cerium (IV) ammonium nitrate can also be used as a nitrating agent $[5,6]$, initiator for radical polymerization reactions [7], and also as a reagent to remove protecting groups [8]. Even though cerium (IV) reagents are milder oxidation reagents than the other metal-based oxidation reagents such as $\mathrm{Mn}(\mathrm{VII})$ and $\mathrm{Cr}(\mathrm{VI})$ salts, they are relatively much less toxic. Because of their high molecular mass, large quantities of cerium (IV) salts are required for stoichiometric reactions. Therefore, indirect and catalytic reactions using $\mathrm{Ce}(\mathrm{IV})$ have been developed $[9,10]$. The main advantage of CAN over other cerium (IV) reagents is its higher solubility in organic solvents. The most popular solvents are (in decreasing order of importance) water, acetonitrile, dichloromethane, THF, and methanol [11]. Often mixtures of these solvents are also used as reaction medium. Other solvents have found only marginal use for this type of reactions.

Being good Michael acceptors, $\alpha, \beta$-unsaturated nitroalkenes are widely applied in organic synthesis [12]. Among various methods reported for their preparation, a method involving the use of CAN provides a practical way to the synthesis of $\alpha, \beta$-unsaturated "nitroalkenes" in goodto-excellent yields [13-23]. Recently polyethylene glycols (PEGs) have been used as catalysts and catalyst supports and also have been found to be an inexpensive, nontoxic, and environmentally friendly reaction medium, which avoid the use of acid or base catalysts. Moreover PEG can be recovered after completion of the reactions and recycled/reused [2429] in another batch. Inspired by the striking features of PEG the author wants to use it as a catalyst by avoiding the use of acid in the present study; namely, ceric ammonium 
TABLE 1: Binding constants of [CAN-PEG] at $303^{\circ} \mathrm{K}$ using Benesi-Hildebrand method.

\begin{tabular}{lcccc}
\hline S. N & PEG & Benesi-Hildebrand equation & $\begin{array}{c}K \\
\mathrm{dm}^{3} / \mathrm{mol}\end{array}$ & $\begin{array}{c}\varepsilon \\
\mathrm{dm}^{3} / \mathrm{mol} / \mathrm{cm}\end{array}$ \\
\hline 1 & PEG-200 & $y=7 E-05 x+0.052$ & 743 & 19.23 \\
2 & PEG-300 & $y=9 E-05 x+0.055$ & 611 & 18.18 \\
3 & PEG-400 & $y=5 E-05 x+0.071$ & 1420 & 14.08 \\
\hline
\end{tabular}

TABLE 2: Activation parameters of cinnamic acid in different PEG media. Units of $k^{\prime \prime}=\mathrm{dm}^{3} \mathrm{~mol}^{-1} \mathrm{~min}^{-1}$.

\begin{tabular}{|c|c|c|c|c|c|c|c|}
\hline Type of PEG & $\begin{array}{c}\text { PEG } \\
\%(\mathrm{~V} / \mathrm{V})\end{array}$ & $\begin{array}{l}k^{\prime \prime} \text { at } \\
300 \mathrm{~K}\end{array}$ & Equation & $R^{2}$ & \multicolumn{2}{|c|}{$\mathrm{kJ} / \mathrm{mol}$} & $\begin{array}{c}\Delta S^{\#} \\
\mathrm{~J} / \mathrm{K} / \mathrm{mol}\end{array}$ \\
\hline \multirow{6}{*}{ PEG-200 } & 0.5 & 0.01 & $y=-13.32 x+34.09$ & 0.999 & 110 & 84.2 & 85.9 \\
\hline & 1.0 & 0.02 & $y=-9.181 x+20.97$ & 0.999 & 76.3 & 83.3 & -23.2 \\
\hline & 2.0 & 0.03 & $y=-8.399 x+18.75$ & 0.995 & 70.0 & 95.5 & -41.7 \\
\hline & 3.0 & 0.04 & $y=-8.625 x+19.84$ & 0.998 & 91.8 & 81.8 & -32.6 \\
\hline & 4.0 & 0.08 & $y=-7.047 x+15.06$ & 0.999 & 58.6 & 55.6 & -72.3 \\
\hline & 5.0 & 0.1 & $y=-8.112 x+19.01$ & 0.995 & 67.5 & 39.7 & 39.5 \\
\hline \multirow{6}{*}{ PEG-300 } & 0.5 & 0.01 & $y=-22.79 x+64.43$ & 0.997 & 189 & 87.6 & 338 \\
\hline & 1.0 & 0.03 & $y=-4.655 x+6.308$ & 0.996 & 38.7 & 82.3 & -145 \\
\hline & 2.0 & 0.04 & $y=-5.385 x+8.930$ & 0.999 & 44.8 & 81.8 & -123 \\
\hline & 3.0 & 0.05 & $y=-2.684 x+0.252$ & 0.998 & 22.4 & 81.0 & -195 \\
\hline & 4.0 & 0.06 & $y=-8.743 x+20.63$ & 0.999 & 72.7 & 80.5 & -20.0 \\
\hline & 5.0 & 0.08 & $y=-7.750 x+17.60$ & 0.999 & 64.5 & 80.0 & -51.3 \\
\hline \multirow{6}{*}{ PEG-400 } & 0.5 & 0.02 & $y=-6.979 x+13.65$ & 0.999 & 58.0 & 83.3 & -84.0 \\
\hline & 1.0 & 0.03 & $y=-6.106 x+11.05$ & 0.997 & 50.8 & 82.5 & -105 \\
\hline & 2.0 & 0.04 & $y=-9.224 x+21.84$ & 0.999 & 76.7 & 81.5 & -16.0 \\
\hline & 3.0 & 0.06 & $y=-8.718 x+20.54$ & 0.999 & 72.5 & 38.0 & -80.5 \\
\hline & 4.0 & 0.07 & $y=-7.054 x+15.38$ & 0.999 & 58.7 & 80.1 & -69.7 \\
\hline & 5.0 & 0.1 & $y=-7.687 x+17.63$ & 0.999 & 64.0 & 79.3 & -51.0 \\
\hline \multirow{6}{*}{ PEG-600 } & 0.5 & 0.02 & $y=-5.421 x+8.550$ & 0.998 & 45.0 & 83.0 & -126 \\
\hline & 1.0 & 0.03 & $y=-4.644 x+6.274$ & 0.999 & 38.6 & 82.2 & -145 \\
\hline & 2.0 & 0.04 & $y=-3.131 x+1.542$ & 0.999 & 26.0 & 81.4 & -184 \\
\hline & 3.0 & 0.05 & $y=-5.555 x+9.813$ & 0.999 & 46.2 & 81.0 & -115 \\
\hline & 4.0 & 0.07 & $y=-3.622 x+3.753$ & 0.998 & 30.1 & 80.0 & -166 \\
\hline & 5.0 & 0.1 & $y=-4.247 x+5.956$ & 0.999 & 35.3 & 80.0 & -148 \\
\hline
\end{tabular}

nitrate (CAN) triggered decarboxylative nitration of $\alpha, \beta$ unsaturated aromatic carboxylic acids. Acetonitrile is used as solvent in order to facilitate kinetic studies.

\section{Experimental Details}

2.1. Materials. Cinnamic acid, ceric ammonium nitrate (CAN), and polyethylene glycols were obtained from SD Fine Chemicals or Loba. Substituted cinnamic acids were prepared by Perkins reaction as cited in the literature $[30,31]$.

\subsection{General Procedure for PEG-Mediated $\beta$-Nitrostyrenes} Synthesis. In a typical solid state synthesis, cinnamic acid (0.01 mol), PEG (0.02 mmol), and CAN (0.012 $\mathrm{mmol})$ are placed in a clean two-necked R. B. flask containing acetonitrile $(\mathrm{MeCN})$ and stirred for certain time. Progress of the reaction is periodically monitored by thin layer chromatography (TLC). After completion, the reaction mixture is treated with $2 \%$ sodium carbonate solution, followed by the addition of "dichloromethane" (DCM) or "dichloroethane" (DCE). The organic layer was separated, dried over $\mathrm{Na}_{2} \mathrm{SO}_{4}$ and evaporated under vacuum, and purified with column chromatography using ethyl acetate : hexane $(3: 7)$ as eluent to get pure product. The products were characterized by IR, 1H-NMR, mass spectra, and physical data with authentic samples and found to agree well with earlier reports (Table 1). The yields of major products are compiled in Table 2.

2.3. Kinetic Method of Following the Reaction. Thermostat was adjusted to desired reaction temperature. Flask containing known amount of ceric ammonium nitrate (CAN) in acetonitrile solvent and another flask containing the substrate $(\alpha, \beta$-unsaturated acid) and suitable amount of PEG solutions were clamped in a thermostatic bath. Reaction was initiated by mixing requisite amount of CAN with the other contents of the reaction vessel. The entire reaction mixture was 
TABLE 3: Activation parameters of crotonic acid in different PEG media. Units of $k^{\prime \prime}=\mathrm{dm}^{3} \mathrm{~mol}^{-1} \mathrm{~min}^{-1}$.

\begin{tabular}{|c|c|c|c|c|c|c|c|}
\hline Type of PEG & $\begin{array}{c}\text { PEG } \\
\%(\mathrm{~V} / \mathrm{V})\end{array}$ & $\begin{array}{l}k^{\prime \prime} \text { at } \\
300 \mathrm{~K}\end{array}$ & Equation & $R^{2}$ & \multicolumn{2}{|c|}{$\mathrm{kJ} / \mathrm{mol}$} & $\begin{array}{c}\Delta S^{\#} \\
\mathrm{~J} / \mathrm{K} / \mathrm{mol}\end{array}$ \\
\hline \multirow{6}{*}{ PEG-200 } & 0.5 & 0.01 & $y=-9.907 x+22.7$ & 0.999 & 82.4 & 85.1 & -9.0 \\
\hline & 1.0 & 0.02 & $y=-6.742 x+12.86$ & 0.999 & 56.0 & 83.3 & -91.0 \\
\hline & 2.0 & 0.03 & $y=-5.069 x+7.688$ & 0.999 & 42.1 & 82.3 & -134 \\
\hline & 3.0 & 0.04 & $y=-6.020 x+11.13$ & 0.999 & 50.0 & 81.5 & 105 \\
\hline & 4.0 & 0.05 & $y=-7.664 x+16.87$ & 0.999 & 64.0 & 81.2 & -57.3 \\
\hline & 5.0 & 0.06 & $y=-7.062 x+14.92$ & 0.999 & 59.0 & 81.0 & -73.5 \\
\hline \multirow{6}{*}{ PEG-300 } & 0.5 & 0.01 & $y=-9.797 x+22.35$ & 0.999 & 81.4 & 85.0 & -12.0 \\
\hline & 1.0 & 0.02 & $y=-8.070 x+17.25$ & 0.999 & 67.0 & 83.2 & -54.2 \\
\hline & 2.0 & 0.04 & $y=-4.233 x+5.180$ & 0.998 & 35.2 & 81.5 & -154 \\
\hline & 3.0 & 0.05 & $y=-6.852 x+14.13$ & 0.999 & 54.6 & 79.0 & -80.0 \\
\hline & 4.0 & 0.06 & $y=-6.564 x+13.36$ & 0.999 & 54.5 & 80.4 & -86.5 \\
\hline & 5.0 & 0.08 & $y=-9.440 x+23.13$ & 0.999 & 78.5 & 80.0 & -5.3 \\
\hline \multirow{6}{*}{ PEG-400 } & 0.5 & 0.01 & $y=-9.759 x+22.23$ & 0.999 & 81.2 & 85.0 & -13.0 \\
\hline & 1.0 & 0.02 & $y=-7.864 x+16.59$ & 0.999 & 65.4 & 83.3 & -57.0 \\
\hline & 2.0 & 0.04 & $y=-4.189 x+5.034$ & 0.999 & 35.0 & 82.0 & -155 \\
\hline & 3.0 & 0.05 & $y=-8.018 x+17.93$ & 0.999 & 67.0 & 81.5 & -48.5 \\
\hline & 4.0 & 0.06 & $y=-5.394 x+9.454$ & 0.999 & 45.0 & 80.7 & -119 \\
\hline & 5.0 & 0.08 & $y=-8.250 x+19.25$ & 0.998 & 68.6 & 80.0 & -37.5 \\
\hline \multirow{6}{*}{ PEG-600 } & 0.5 & 0.01 & $y=-9.630 x+21.81$ & 0.998 & 80.0 & 85.0 & -16.3 \\
\hline & 1.0 & 0.02 & $y=-5.541 x+8.852$ & 0.998 & 46.0 & 83.2 & -123 \\
\hline & 2.0 & 0.03 & $y=-5.256 x+8.404$ & 0.999 & 44.0 & 82.3 & -128 \\
\hline & 3.0 & 0.04 & $y=-5.421 x+9.243$ & 0.998 & 45.0 & 81.2 & -120 \\
\hline & 4.0 & 0.06 & $y=-4.492 x+6.466$ & 0.997 & 37.4 & 80.5 & -144 \\
\hline & 5.0 & 0.09 & $y=-6.254 x+12.73$ & 0.999 & 52.0 & 80.0 & -91.8 \\
\hline
\end{tabular}

UV-Visible spectra of CAN in presence and absence of PEG

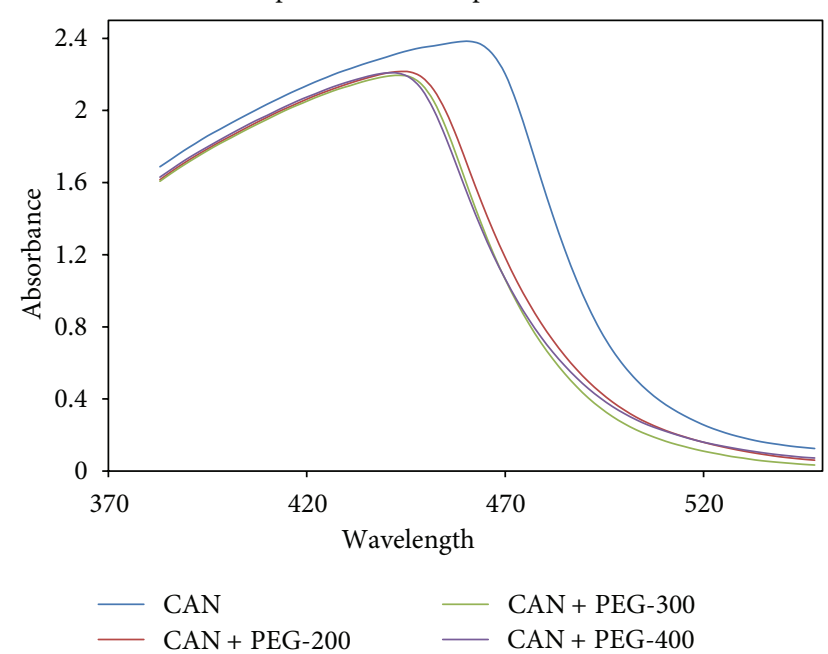

FIGURE 1: UV-Visible spectra of CAN in presence and absence of $\mathrm{PEG}$ in $\mathrm{MeCN}$ medium.

mixed thoroughly. Flask was coated black from the outside to prevent photochemical effects. Aliquots of the reaction

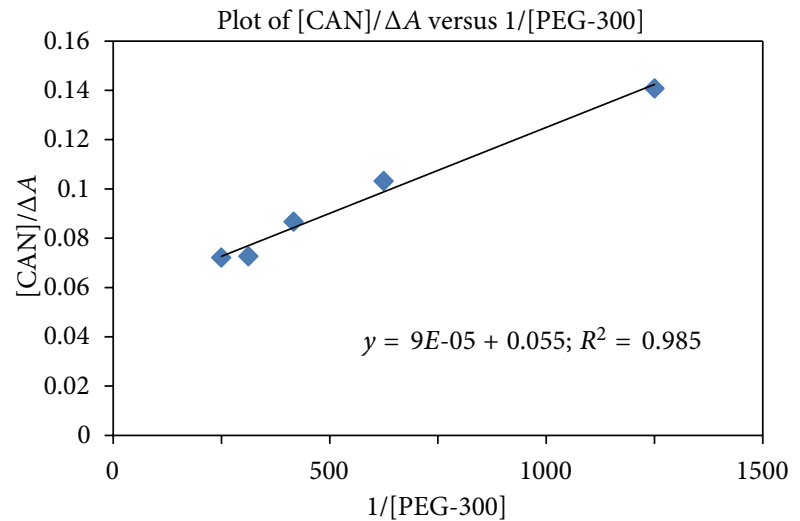

FIGURE 2: Benesi-Hildebrand plot of PEG-300 interaction with CAN.

mixture were withdrawn into a cuvette and placed in the cell compartment of the laboratory visible spectrophotometer. Cell compartment was provided with an inlet and an outlet for circulation of thermostatic liquid at a desired temperature. The CAN content could be estimated from the previously constructed calibration curve showing absorbance $(A)$ versus $[\mathrm{CAN}]$. Absorbance values were in agreement with each other with an accuracy of $\pm 3 \%$ error. 
TABLE 4: Activation parameters of methoxy cinnamic acid in different PEG media. Units of $k^{\prime \prime}=\mathrm{dm}^{3} \mathrm{~mol}^{-1} \mathrm{~min}^{-1}$.

\begin{tabular}{|c|c|c|c|c|c|c|c|}
\hline Type of PEG & $\begin{array}{c}\text { PEG } \\
\%(\mathrm{~V} / \mathrm{V})\end{array}$ & $\begin{array}{l}k^{\prime \prime} \text { at } \\
300 \mathrm{~K}\end{array}$ & Equation & $R^{2}$ & $\Delta H^{\#}$ & $\Delta G^{\#}$ & $\begin{array}{c}\Delta S^{\#} \\
\mathrm{~J} / \mathrm{K} / \mathrm{mol}\end{array}$ \\
\hline \multirow{6}{*}{ PEG-200 } & 0.5 & 0.01 & $y=-12.85 x+32.55$ & 0.996 & 107 & 85.0 & 73.0 \\
\hline & 1.0 & 0.02 & $y=-13.90 x+36.01$ & 0.998 & 115 & 84.4 & 102 \\
\hline & 2.0 & 0.04 & $y=-6.038 x+11.21$ & 0.999 & 50.2 & 81.50 & -104 \\
\hline & 3.0 & 0.06 & $y=-5.027 x+8.448$ & 0.999 & 42.0 & 80.2 & -127 \\
\hline & 4.0 & 0.08 & $y=-3.844 x+4.748$ & 0.999 & 32.0 & 80.0 & -158 \\
\hline & 5.0 & 0.12 & $y=-4.403 x+6.945$ & 0.999 & 37.0 & 79.0 & -140 \\
\hline \multirow{6}{*}{ PEG-300 } & 0.5 & 0.01 & $y=-10.36 x+14.11$ & 0.997 & 86.0 & 35.3 & 110 \\
\hline & 1.0 & 0.03 & $y=-3.532 x+2.653$ & 0.999 & 46.0 & 98.5 & -175 \\
\hline & 2.0 & 0.04 & $y=-5.385 x+8.930$ & 0.959 & 45.0 & 82.0 & -123 \\
\hline & 3.0 & 0.05 & $y=-2.423 x-0.582$ & 0.999 & 20.2 & 78.0 & -192 \\
\hline & 4.0 & 0.06 & $y=-8.743 x+20.63$ & 0.999 & 73.0 & 81.0 & -26.0 \\
\hline & 5.0 & 0.08 & $y=-7.965 x+18.28$ & 0.999 & 66.2 & 80.0 & -46.0 \\
\hline \multirow{6}{*}{ PEG-400 } & 0.5 & 0.02 & $y=-7.638 x+15.83$ & 0.999 & 63.5 & 83.3 & -66.0 \\
\hline & 1.0 & 0.03 & $y=-10.01 x+24.07$ & 0.999 & 83.2 & 77.0 & 21.3 \\
\hline & 2.0 & 0.04 & $y=-9.756 x+23.58$ & 0.999 & 81.0 & 81.4 & -2.0 \\
\hline & 3.0 & 0.06 & $y=-8.022 x+18.16$ & 0.999 & 67.0 & 80.0 & -41.0 \\
\hline & 4.0 & 0.08 & $y=-6.000 x+11.86$ & 0.999 & 50.0 & 80.0 & -99.0 \\
\hline & 5.0 & 0.10 & $y=-9.302 x+22.89$ & 0.999 & 77.3 & 79.5 & -7.3 \\
\hline \multirow{6}{*}{ PEG-600 } & 0.5 & 0.02 & $y=-7.936 x+16.83$ & 0.999 & 66.0 & 83.3 & -58.0 \\
\hline & 1.0 & 0.03 & $y=-5.955 x+10.61$ & 0.998 & 49.5 & 82.3 & -110 \\
\hline & 2.0 & 0.05 & $y=-4.894 x+7.616$ & 0.999 & 41.0 & 81.3 & -134 \\
\hline & 3.0 & 0.06 & $y=-6.259 x+12.33$ & 0.999 & 52.0 & 80.5 & -95.0 \\
\hline & 4.0 & 0.08 & $y=-5.659 x+10.63$ & 0.999 & 47.0 & 38.72 & -110 \\
\hline & 5.0 & 0.1 & $y=-6.719 x+14.05$ & 0.998 & 56.0 & 80.3 & -81.0 \\
\hline
\end{tabular}

2.4. [CAN-PEG] Binding Study. Spectrophotometric studies were performed in order to throw light on CAN binding with PEG (polyethylene glycol). UV-Visible spectrum of $\mathrm{CAN}$ in $\mathrm{MeCN}$ indicated a band at $459 \mathrm{~nm}$. It underwent a hypsochromic shift (blue shift) of about 17 to $18 \mathrm{~nm}$ (band-shifted from 441 to $442 \mathrm{~nm}$ ) in presence of PEG (Figure 1), followed by hypochromic shift. This observation indicates the formation of [CAN-PEG] complex. The CANPEG binding constants $(K)$ were evaluated by the method of Benesi-Hildebrand [32]. When the equilibrium for molecular complexation is taken into consideration,

$$
\mathrm{PEG}+\mathrm{CAN} \stackrel{K}{\rightleftharpoons} \mathrm{C}
$$

The equilibrium constant $K=[\mathrm{C}] /[\mathrm{CAN}][\mathrm{PEG}]$, where [CAN], [PEG], and [C] are equilibrium concentrations of acceptor (CAN), donor (PEG), and complex, respectively. If $[\mathrm{CAN}]_{0}$ and $[\mathrm{PEG}]_{0}$ represent initial concentrations of CAN and $P E G$, respectively, then

$$
K=\frac{[\mathrm{C}]}{\left([\mathrm{CAN}]_{0}-[\mathrm{C}]\right)\left([\mathrm{PEG}]_{0}-[\mathrm{C}]\right)}
$$

Under condition that $[\mathrm{PEG}]_{0} \gg[\mathrm{CAN}]_{0} \Rightarrow[\mathrm{PEG}]_{0} \gg[\mathrm{C}]$ and $\left([\mathrm{PEG}]_{0}-[\mathrm{C}]\right) \sim[\mathrm{PEG}]_{0}$

$$
\begin{aligned}
K & =\frac{[\mathrm{C}]}{\left([\mathrm{CAN}]_{0}-[\mathrm{C}]\right)[\mathrm{PEG}]_{0}} \\
& \Longrightarrow K=\frac{[\mathrm{C}]}{\left([\mathrm{CAN}]_{0}[\mathrm{PEG}]_{0}-[\mathrm{C}][\mathrm{PEG}]_{0}\right)}, \\
{[\mathrm{C}]\left(1+K[\mathrm{PEG}]_{0}\right) } & =K[\mathrm{CAN}]_{0}[\mathrm{PEG}]_{0} \\
& \Longrightarrow[\mathrm{C}]=\frac{K[\mathrm{CAN}]_{0}[\mathrm{PEG}]_{0}}{\left(1+K[\mathrm{PEG}]_{0}\right)}
\end{aligned}
$$

But according to Lambert-Beer's law absorbance $(A)=\epsilon c l$.

In the previous equations, $l$ is path length, $A$ is absorbance, $\epsilon$ is the molar extinction coefficient, and $K$ is formation constant of the complex, respectively,

$$
[\mathrm{C}]=\frac{A}{\epsilon l}=\frac{K[\mathrm{CAN}]_{0}[\mathrm{PEG}]_{0}}{\left(1+K[\mathrm{PEG}]_{0}\right)} \text {. }
$$

According to this plot of absorbance $(A)$ as a function of donor concentration $\left([\mathrm{PEG}]_{0}\right)$ the plot indicated a linear increase in the value of $(A)$ with an increase in $\left([\mathrm{PEG}]_{0}\right)$ with 
TABLE 5: Activation parameters of nitrocinnamic acid in different PEG media. Units of $k^{\prime \prime}=\mathrm{dm}^{3} \mathrm{~mol}^{-1} \mathrm{~min}^{-1}$.

\begin{tabular}{|c|c|c|c|c|c|c|c|}
\hline Type of PEG & $\begin{array}{c}\text { PEG } \\
\%(\mathrm{~V} / \mathrm{V})\end{array}$ & $\begin{array}{l}k^{\prime \prime} \text { at } \\
300 \mathrm{~K}\end{array}$ & Equation & $R^{2}$ & $\Delta H^{\#}$ & $\Delta G^{\#}$ & $\begin{array}{c}\Delta S^{\#} \\
\mathrm{~J} / \mathrm{K} / \mathrm{mol}\end{array}$ \\
\hline \multirow{6}{*}{ PEG-200 } & 0.5 & 0.01 & $y=-12.00 x+29.73$ & 0.999 & 100 & 85.0 & 50.0 \\
\hline & 1.0 & 0.02 & $y=-10.87 x+26.63$ & 0.999 & 90.3 & 83.1 & 24.0 \\
\hline & 2.0 & 0.04 & $y=-4.711 x+6.807$ & 0.999 & 39.2 & 37.05 & -141 \\
\hline & 3.0 & 0.06 & $y=-5.506 x+9.842$ & 0.999 & 46.0 & 38.04 & -116 \\
\hline & 4.0 & 0.07 & $y=-5.012 x+8.347$ & 0.999 & 42.0 & 38.40 & -128 \\
\hline & 5.0 & 0.09 & $y=-3.923 x+4.963$ & 0.998 & 33.0 & 38.99 & -156 \\
\hline \multirow{6}{*}{ PEG-300 } & 0.5 & 0.01 & $y=-9.764 x+22.25$ & 0.999 & 81.2 & 85.0 & -13.0 \\
\hline & 1.0 & 0.02 & $y=-7.861 x+16.58$ & 0.999 & 65.4 & 83.3 & -60.0 \\
\hline & 2.0 & 0.04 & $y=-4.270 x+5.297$ & 0.999 & 35.5 & 37.0 & -153 \\
\hline & 3.0 & 0.05 & $y=-6.852 x+14.13$ & 0.999 & 57.0 & 81.0 & -80.0 \\
\hline & 4.0 & 0.06 & $y=-6.545 x+13.3$ & 0.998 & 54.4 & 81.2 & -89.5 \\
\hline & 5.0 & 0.08 & $y=-9.042 x+21.84$ & 0.999 & 75.1 & 80.0 & -16.0 \\
\hline \multirow{6}{*}{ PEG-400 } & 0.5 & 0.02 & $y=-7.717 x+16.10$ & 0.977 & 64.1 & 83.2 & -64.0 \\
\hline & 1.0 & 0.03 & $y=-5.321 x+8.612$ & 0.999 & 44.2 & 82.0 & -126 \\
\hline & 2.0 & 0.04 & $y=-8.683 x+20.01$ & 0.999 & 72.1 & 81.4 & -31.2 \\
\hline & 3.0 & 0.06 & $y=-8.718 x+20.54$ & 0.999 & 72.4 & 80.4 & -27.0 \\
\hline & 4.0 & 0.07 & $y=-7.947 x+18.23$ & 0.998 & 66.0 & 80.0 & -46.0 \\
\hline & 5.0 & 0.10 & $y=-7.687 x+17.63$ & 0.999 & 64.0 & 79.3 & -51.0 \\
\hline \multirow{6}{*}{ PEG-600 } & 0.5 & 0.01 & $y=-9.635 x+21.82$ & 0.998 & 80.1 & 85.0 & -16.2 \\
\hline & 1.0 & 0.02 & $y=-8.534 x+18.80$ & 0.997 & 71.0 & 83.4 & -41.3 \\
\hline & 2.0 & 0.04 & $y=-4.071 x+4.648$ & 0.999 & 34.0 & 82.0 & -159 \\
\hline & 3.0 & 0.06 & $y=-4.703 x+7.167$ & 0.999 & 39.1 & 80.5 & -138 \\
\hline & 4.0 & 0.08 & $y=-3.635 x+3.891$ & 0.999 & 30.2 & 80.0 & -165 \\
\hline & 5.0 & 0.10 & $y=-5.600 x+10.65$ & 0.999 & 46.5 & 79.2 & -109 \\
\hline
\end{tabular}

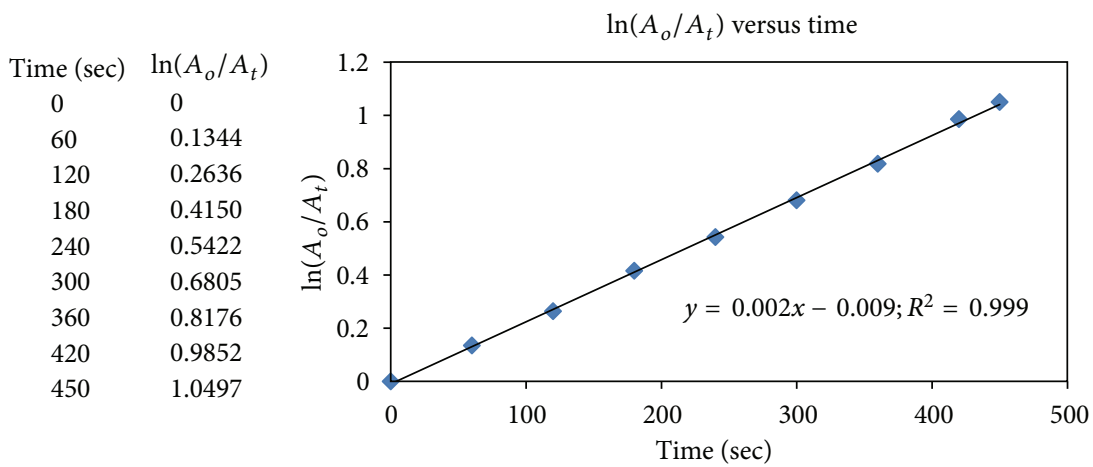

Figure 3: Pseudo first order plot of cinnamic acid $\left(\ln \left(A_{o} / A_{t}\right)\right.$ versus time $)$ at $323 \mathrm{~K}$. $[\mathrm{CA}]=1.00 \times 10^{-2} \mathrm{~mol} \mathrm{dm}^{-3}$; $[\mathrm{CAN}]=4.00 \times$ $10^{-3} \mathrm{~mol} \mathrm{dm}^{-3}$; [PEG-300] $=9.99 \times 10^{-1} \mathrm{~mol} \mathrm{dm}^{-3}$.

a limiting value at its higher concentrations. Further, taking the reciprocals to the previous equation, it rearranges to

$$
\left(\frac{[\mathrm{CAN}]_{0}}{A l}\right)=\left(\frac{1}{K[\mathrm{PEG}]_{0} \epsilon}\right)+\left(\frac{1}{\epsilon}\right) \text {. }
$$

For one $\mathrm{cm}$ path length, the aforementioned equation can be written as

$$
\left(\frac{[\mathrm{CAN}]_{0}}{A}\right)=\left(\frac{1}{K[\mathrm{PEG}]_{0} \epsilon}\right)+\left(\frac{1}{\epsilon}\right)
$$

But the absorbance of CAN and [CAN-PEG] absorbs in the same region significantly; therefore the observed absorbance (A) could be written as

$$
A=A_{(\mathrm{CAN})}+A_{(\text {Complex })}
$$

Now the true absorbance of [CAN-PEG] adduct is the difference between observed absorbance and absorbance of CAN:

$$
\Longrightarrow A_{(\text {Complex })}=\Delta A=A \sim A_{(\mathrm{CAN})} .
$$


TABLE 6: Activation parameters of acrylic acid in different PEG media. Units of $k^{\prime \prime}=\mathrm{dm}^{3} \mathrm{~mol}^{-1} \mathrm{~min}^{-1}$.

\begin{tabular}{|c|c|c|c|c|c|c|c|}
\hline \multirow{2}{*}{ Type of PEG } & \multirow{2}{*}{$\begin{array}{c}\text { PEG } \\
\%(\mathrm{~V} / \mathrm{V})\end{array}$} & \multirow{2}{*}{$\begin{array}{l}k^{\prime \prime} \text { at } \\
300 \mathrm{~K}\end{array}$} & \multirow{2}{*}{ Equation } & \multirow{2}{*}{$R^{2}$} & $\Delta H^{\#}$ & $\Delta G^{\#}$ & \multirow{2}{*}{$\begin{array}{c}\Delta S^{\#} \\
\mathrm{~J} / \mathrm{K} / \mathrm{mol}\end{array}$} \\
\hline & & & & & \multicolumn{2}{|c|}{$\mathrm{kJ} / \mathrm{mol}$} & \\
\hline \multirow{6}{*}{ PEG-200 } & 0.5 & 0.01 & $y=-11.85 x+29.21$ & 0.999 & 98.5 & 85.0 & 45.3 \\
\hline & 1.0 & 0.02 & $y=-8.384 x+18.31$ & 0.998 & 70.0 & 84.0 & -45.3 \\
\hline & 2.0 & 0.03 & $y=-7.860 x+16.98$ & 0.998 & 65.3 & 82.2 & -57.0 \\
\hline & 3.0 & 0.04 & $y=-8.164 x+18.27$ & 0.997 & 68.0 & 82.0 & -47.0 \\
\hline & 4.0 & 0.06 & $y=-6.581 x+13.42$ & 0.999 & 55.0 & 81.0 & -86.0 \\
\hline & 5.0 & 0.08 & $y=-6.061 x+11.96$ & 0.998 & 51.0 & 80.5 & -98.2 \\
\hline \multirow{6}{*}{ PEG-300 } & 0.5 & 0.01 & $y=-11.85 x+29.21$ & 0.999 & 98.5 & 85.0 & 46.0 \\
\hline & 1.0 & 0.02 & $y=-11.43 x+28.49$ & 0.998 & 95.0 & 83.2 & 39.3 \\
\hline & 2.0 & 0.03 & $y=-10.67 x+26.25$ & 0.997 & 89.0 & 83.0 & 21.0 \\
\hline & 3.0 & 0.04 & $y=-9.163 x+21.61$ & 0.999 & 76.2 & 82.0 & -18.0 \\
\hline & 4.0 & 0.05 & $y=-6.125 x+11.72$ & 0.999 & 51.0 & 81.0 & -100 \\
\hline & 5.0 & 0.07 & $y=-5.443 x+9.792$ & 0.998 & 45.3 & 80.2 & -116 \\
\hline \multirow{6}{*}{ PEG-400 } & 0.5 & 0.02 & $y=-8.314 x+18.07$ & 0.997 & 69.2 & 83.4 & -47.4 \\
\hline & 1.0 & 0.03 & $y=-9.364 x+21.98$ & 0.998 & 78.0 & 82.5 & -15.0 \\
\hline & 2.0 & 0.04 & $y=-9.756 x+23.58$ & 0.999 & 81.2 & 82.0 & -1.6 \\
\hline & 3.0 & 0.06 & $y=-7.889 x+17.76$ & 0.997 & 66.0 & 81.0 & -50.0 \\
\hline & 4.0 & 0.08 & $y=-6.573 x+13.69$ & 0.998 & 55.0 & 80.2 & -84.0 \\
\hline & 5.0 & 0.10 & $y=-8.507 x+20.35$ & 0.999 & 71.0 & 80.0 & -28.4 \\
\hline \multirow{6}{*}{ PEG-600 } & 0.5 & 0.01 & $y=-9.947 x+22.82$ & 0.998 & 83.0 & 85.4 & -8.0 \\
\hline & 1.0 & 0.02 & $y=-6.653 x+12.57$ & 0.997 & 55.4 & 83.3 & -93.0 \\
\hline & 2.0 & 0.03 & $y=-6.334 x+11.88$ & 0.997 & 53.0 & 83.0 & -99.0 \\
\hline & 3.0 & 0.05 & $y=-2.362 x-0.780$ & 0.998 & 20.0 & 77.4 & -191 \\
\hline & 4.0 & 0.07 & $y=-3.078 x+1.893$ & 0.998 & 26.0 & 80.5 & -182 \\
\hline & 5.0 & 0.09 & $y=-4.666 x+7.455$ & 0.998 & 39.0 & 80.0 & -135 \\
\hline
\end{tabular}

$\begin{array}{cl}\text { Time }(\mathrm{sec}) & \ln \left(A_{o} / A_{t}\right) \\ 0 & 0 \\ 5 & 0.068 \\ 10 & 0.1431 \\ 15 & 0.2025 \\ 25 & 0.34015 \\ 30 & 0.4054 \\ 35 & 0.4834 \\ 40 & 0.5679 \\ 45 & 0.6286 \\ 55 & 0.7621 \\ 65 & 0.9162\end{array}$

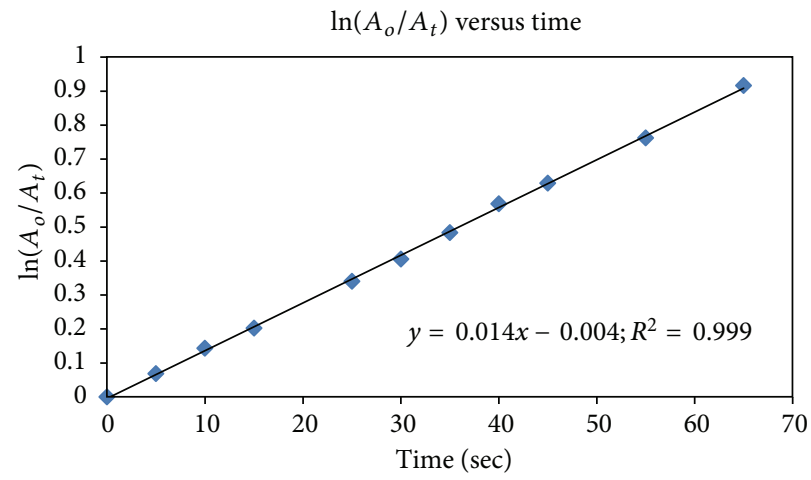

FIGURE 4: Pseudo first order plot of acrylic acid $\left(\ln \left(A_{o} / A_{t}\right)\right.$ versus time $)$ at $315 \mathrm{~K}$. $[\mathrm{AA}]=1.00 \times 10^{-2} \mathrm{~mol} \mathrm{dm}^{-3} ;[\mathrm{CAN}]=4.00 \times 10^{-3} \mathrm{~mol} \mathrm{dm}{ }^{-3}$; $\left[\right.$ PEG-300] $=13.32 \times 10^{-2} \mathrm{~mol} \mathrm{dm}{ }^{-3}$.

Therefore, a plot of $\left([\mathrm{CAN}]_{0} / \Delta A\right)$ versus $1 /[\mathrm{PEG}]_{0}$ should give a straight line according to the previous equation. These plots (Figure 2) have been realized in the present study. Formation constant $(K)$ has been calculated from the ratio of intercept to slope, while inverse of the intercept gave molar extinction coefficient $(\epsilon)$ and is represented in Table 1.

\section{Results and Discussion}

3.1. Determination of the Order of Reaction. General form of the rate law for a nitrodecarboxylation (nitro-Hunsdiecker reaction) reaction could be represented by considering the following general scheme:

$$
\mathrm{CAN}+\mathrm{S} \longrightarrow \text { Products }
$$

where $\mathrm{CAN}=$ ceric ammonium nitrate and $\mathrm{S}=\alpha, \beta$ unsaturated carboxylic acid:

$$
V=k_{1}[\mathrm{CAN}]^{x}[\mathrm{CAN}]^{y} .
$$

Reactions were conducted under two different conditions. Under pseudo first order conditions [CAN] $\gg[\mathrm{CAN}]$, 


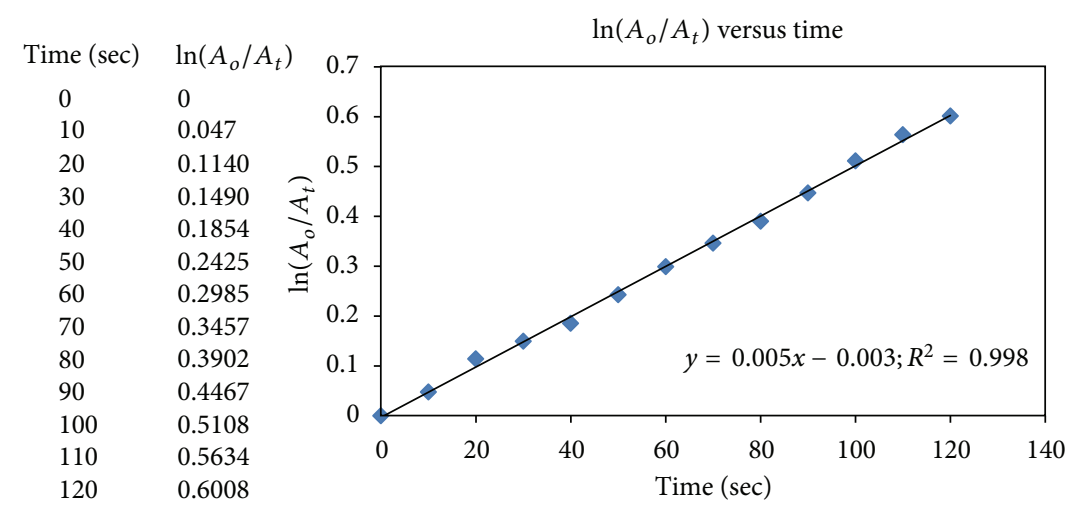

FIGURE 5: Pseudo first order plot of nitrocinnamic acid $\left(\ln \left(A_{o} / A_{t}\right)\right.$ versus time $)$ at $315 \mathrm{~K}$. $[\mathrm{NCA}]=1.00 \times 10^{-2} \mathrm{~mol} \mathrm{dm}^{-3}$; $[\mathrm{CAN}]=4.00 \times$ $10^{-3} \mathrm{~mol} \mathrm{dm}^{-3}$; [PEG-400] $=1.25 \times 10^{-1} \mathrm{~mol} \mathrm{dm}^{-3}$.

$\begin{array}{cc}\text { Time }(\min ) & 1 / A_{t} \\ 0 & 1.1543 \\ 10 & 1.8408 \\ 20 & 2.3255 \\ 30 & 2.9727 \\ 40 & 3.6433 \\ 50 & 4.2761 \\ 70 & 4.8236 \\ 80 & 5.4823 \\ 90 & 6.1428\end{array}$

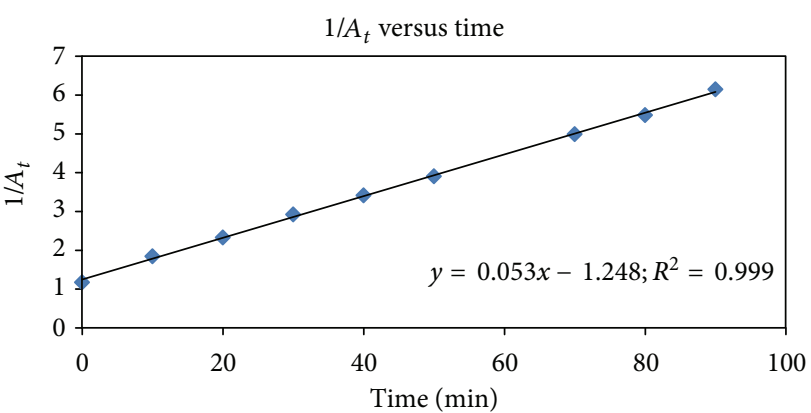

FIGURE 6: Second order kinetic plot of cinnamic acid $\left(1 / A_{t}\right.$ versus Time $)$ at $310 \mathrm{~K}$. $[\mathrm{CA}]=4.00 \times 10^{-2} \mathrm{~mol} \mathrm{dm}^{-3} ;[\mathrm{CAN}]=4.00 \times 10^{-2} \mathrm{~mol} \mathrm{dm}^{-3}$; $\left[\right.$ PEG-200] $=5.00 \times 10^{-1} \mathrm{~mol} \mathrm{dm}^{-3}$.

plots of $\ln \left(A_{o} / A_{t}\right)$ versus time were straight lines with a negative slope indicating order $(x)$ with respect to [CAN] to be unity. As typical examples pseudo first order plots are shown in Figures 3, 4, and 5. First order rate constant $\left(k^{\prime}\right)$ was obtained from the slopes of line plots cited $\left(k^{\prime}\right)$.

The reaction is also conducted under second order conditions with equal concentrations of $[\mathrm{CAN}]_{0}=[\mathrm{CAN}]_{0}$. Kinetic plots of $[1 /(a-x)]$ or $\left[1 /\left(A_{t}\right)\right]$ versus time (Figures 6,7 , and 8 ) have been found to be linear with a positive gradient and definite intercept on ordinate (vertical axis), indicating overall second order kinetics.

In the present study, kinetic data have been collected at three to four different temperatures within the range of 300-315 K. Activation parameters such as $\Delta H^{\#}$ and $\Delta S^{\#}$ have been evaluated from Eyring's plots (Figures 9 and 10). Free energy of activation $\left(\Delta G^{\#}\right)$ is obtained from Gibbs-Helmholtz equation:

$$
\Delta G^{\#}=\Delta H^{\#}-T \Delta S^{\#}
$$

\subsection{Mechanism of Nitrodecarboxylation of Unsaturated Acids} by $\mathrm{Ce}(I V)$ Nitrate $(C A N)$ in MeCN Medium. In order to gain an insight into the mechanistic aspects of CAN-CA reaction in $\mathrm{MeCN}$ medium, the knowledge of distribution of CAN species in $\mathrm{HNO}_{3}$ medium could be useful. In $\mathrm{HNO}_{3} \mathrm{Ce}(\mathrm{IV})$ mainly exists as $\mathrm{Ce}\left(\mathrm{NO}_{3}\right)_{6}{ }^{2-}, \mathrm{Ce}\left(\mathrm{NO}_{3}\right)_{5}{ }^{-}, \mathrm{Ce}(\mathrm{OH})\left(\mathrm{NO}_{3}\right)_{4}{ }^{-}$,
$\mathrm{Ce}\left(\mathrm{NO}_{3}\right)_{4}$, which could arise from the following equilibria as cited in the literature reports [33-36]:

$$
\begin{aligned}
& \mathrm{Ce}\left(\mathrm{NO}_{3}\right)_{4}+\mathrm{NO}_{3}{ }^{-} \rightleftharpoons \mathrm{Ce}\left(\mathrm{NO}_{3}\right)_{5}{ }^{-} \\
& \mathrm{Ce}\left(\mathrm{NO}_{3}\right)_{5}{ }^{-}+\mathrm{NO}_{3}{ }^{-} \rightleftharpoons \mathrm{Ce}\left(\mathrm{NO}_{3}\right)_{6}{ }^{2-} \\
& \mathrm{Ce}\left(\mathrm{NO}_{3}\right)_{4}+\mathrm{H}_{2} \mathrm{O} \rightleftharpoons \mathrm{Ce}(\mathrm{OH})\left(\mathrm{NO}_{3}\right)_{4}{ }^{-}+\mathrm{H}^{+}
\end{aligned}
$$

But the CAN species in the present study could be entirely different because the reactions are conducted in $\mathrm{MeCN}$ medium, which is large excess over [CAN]. MeCN may penetrate into the coordination spheres of $\mathrm{Ce}(\mathrm{IV})$ and form solvated CAN species according to the following equilibrium:

$$
\mathrm{Ce}\left(\mathrm{NO}_{3}\right)_{4}+2 \mathrm{ACN} \rightleftharpoons\left[\mathrm{Ce}\left(\mathrm{NO}_{3}\right)_{4}(\mathrm{ACN})_{2}\right]
$$

When unsaturated acid (USA) is added to the reaction mixture containing solvated CAN, it may form [Ce(IV) carboxylate] precursor which dissociates and releases $\mathrm{HNO}_{3}$ in situ. The $\mathrm{HNO}_{3}$ thus released in turn may combine with another solvated CAN to form nitronium ion $\left(\mathrm{NO}_{2}{ }^{+}\right)$which 


$$
\begin{array}{cccc}
\mathrm{H}-\left(\mathrm{OCH}_{2}-\mathrm{CH}_{2}\right)_{n}-\mathrm{OH}+\left[\mathrm{Ce}\left(\mathrm{NO}_{3}\right)_{4}(\mathrm{ACN})_{2}\right] & \stackrel{K}{\rightleftharpoons}\left[\mathrm{H}-\left(\mathrm{OCH}_{2}-\mathrm{CH}_{2}\right)_{n}-\mathrm{O}-\mathrm{Ce}\left(\mathrm{NO}_{3}\right)_{3}(\mathrm{ACN})_{2}\right] \\
(\mathrm{PEG}) & (\mathrm{CAN}) & -\mathrm{HNO}_{3} & {[\mathrm{PEG}-\mathrm{CAN}]}
\end{array}
$$

SCHEME 1

$$
\mathrm{CAN}+\mathrm{PEG} \stackrel{K}{\rightleftharpoons}[\mathrm{PEG}-\mathrm{CAN}]
$$

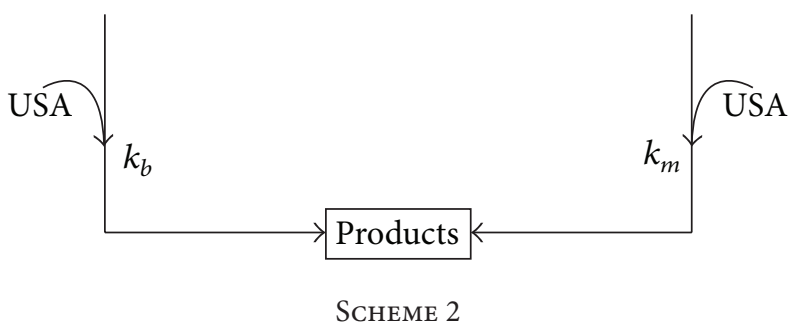

finally reacts with coordinated USA to afford $\beta$-nitrostyrene as shown in the following sequence of steps:

$$
\begin{aligned}
\mathrm{RCH} & =\mathrm{CH}-\mathrm{COOH}+\left[\mathrm{Ce}\left(\mathrm{NO}_{3}\right)_{4}\left(\mathrm{CH}_{3} \mathrm{CHN}\right)_{2}\right] \\
& \rightleftharpoons\left[\mathrm{Ce}\left(\mathrm{NO}_{3}\right)_{4}(\mathrm{ACN})(\mathrm{RCH}=\mathrm{CH}-\mathrm{COOH})\right]
\end{aligned}
$$$$
\text { Precursor } \stackrel{\text { (Slow) }}{\longrightarrow} \mathrm{HNO}_{3}
$$$$
+\left[\mathrm{Ce}\left(\mathrm{NO}_{3}\right)_{3}(\mathrm{ACN})(\mathrm{RCH}=\mathrm{CH}-\mathrm{COOH})\right]
$$$$
\mathrm{HNO}_{3}+\left[\mathrm{Ce}\left(\mathrm{NO}_{3}\right)_{4}(\mathrm{ACN})_{2}\right]
$$$$
\longrightarrow \mathrm{NO}_{2}{ }^{+}+\mathrm{NO}_{3}{ }^{-}+\left[\mathrm{Ce}\left(\mathrm{NO}_{3}\right)_{3}(\mathrm{OH})(\mathrm{ACN})_{2}\right]
$$$$
\mathrm{NO}_{2}{ }^{+}+\left[\mathrm{Ce}\left(\mathrm{NO}_{3}\right)_{3}(\mathrm{ACN})(\mathrm{RCH}=\mathrm{CH}-\mathrm{COOH})\right]
$$$$
\longrightarrow \mathrm{RCH}=\mathrm{CHNO}_{2}+\mathrm{CO}_{2}+\left[\mathrm{Ce}\left(\mathrm{NO}_{3}\right)_{3}(\mathrm{ACN})\right]^{+}
$$$$
\left[\mathrm{Ce}\left(\mathrm{NO}_{3}\right)_{3}(\mathrm{ACN})\right]^{+}+\mathrm{NO}_{3}{ }^{-}+\mathrm{ACN}
$$$$
\longrightarrow\left[\mathrm{Ce}\left(\mathrm{NO}_{3}\right)_{4}(\mathrm{ACN})_{2}\right]
$$

3.3. Mechanism of Nitrodecarboxylation in PEG Media. Progress of the reaction has been studied in the presence of a set of polyoxyethylene compounds (PEGs) with varied molecular weights ranging from 200 to 600 units, and it was found that the reaction is enhanced remarkably in all PEGs. Reaction times were reduced from $24 \mathrm{hrs}$ to few hours. The catalytic activity was found to be in the increasing order PEG$300>$ PEG-400 > PEG-600 > PEG-200. Further, it is also interesting to note that the absorbance of solvated Ce(IV) species is increased when PEG is added to [CAN] solution. This observation may indicate that solvated [CAN] species could bind with PEG to form PEG supported CAN species according to the equilibrium shown in Scheme 1.
The plots of $k_{m}$ (rate constant of PEG reaction) versus $C_{\mathrm{PEG}}$ (concentration of PEG) indicated a rate maxima nearly in the vicinity of $1.50 \mathrm{~mol} \mathrm{dm}^{-3} \mathrm{PEG}-200,0.99 \mathrm{~mol} \mathrm{dm}^{-3}$ PEG-300, $0.99 \mathrm{~mol} \mathrm{dm}^{-3}$ PEG-400, $0.500 \mathrm{~mol} \mathrm{dm}^{-3}$ PEG600. Efforts have been made to interpret PEG effect on the mechanism of CAN-USA reaction. Mechanism of PEGmediated reactions was explained in the lines of micellar catalysis because PEG resembles the structure of Triton-X. The first and widely applied model is that of Menger and Portnoy [37] which closely resembles that of an enzymatic Catalysis. According to this model, formation of PEG bound reagent (PEG-CAN) could occur in the preequilibrium step due to the interaction of Ce(IV) with PEG. The complex thus formed may possess higher or lower reactivity to give products. A general mechanism is proposed by considering the bulk phase and micellar phase reactions as shown in Scheme 2, where $k_{m}$ and $k_{0}$ or $\left(k_{b}\right)$ represents rate constants for PEG and bulk phases, respectively, and $K$ is the [PEGCAN] binding constant. For the previous mechanism, rate law could be derived according to the following sequence of steps in the lines of micellar catalyzed reactions.

Considering the total concentration of $\left(C_{s}\right)$ as the algebraic sum of free species and PEG bound substrate complex (CAN-PEG) species,

$$
C_{S}=[\mathrm{CAN}]+[\mathrm{CAN}-\mathrm{PEG}] .
$$

From micelle-substrate binding equilibrium,

$$
\begin{aligned}
K & =\frac{[\mathrm{CAN}-\mathrm{PEG}]}{[\mathrm{PEG}][\mathrm{CAN}]}, \text { or } \\
{[\mathrm{CAN}] } & =\frac{[\mathrm{CAN}-\mathrm{PEG}]}{K[\mathrm{PEG}]} .
\end{aligned}
$$




$\begin{array}{cc}\text { Time }(\mathrm{min}) & 1 / A_{t} \\ 0 & 1.3457 \\ 10 & 1.5518 \\ 20 & 1.8230 \\ 30 & 2.0408 \\ 40 & 2.2222 \\ 50 & 2.5000 \\ 60 & 2.8027 \\ 70 & 3.0303 \\ 80 & 3.3333 \\ 90 & 3.5037 \\ 100 & 3.7454\end{array}$

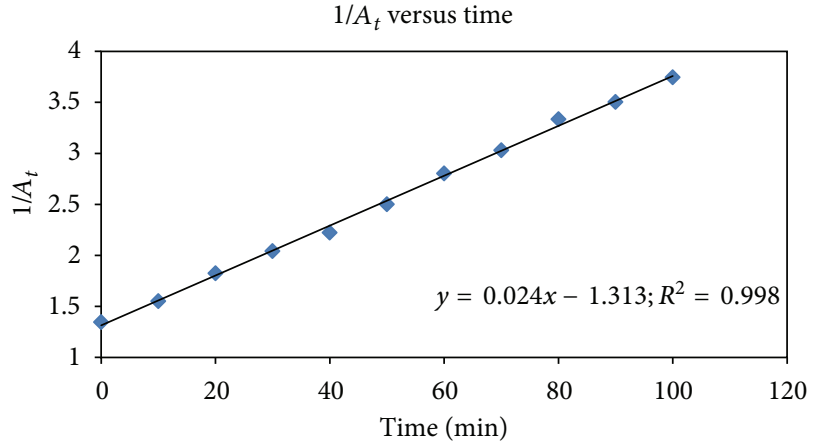

Figure 7: Second order kinetic plot of acrylic acid $\left(1 / A_{t}\right.$ versus Time) at $310 \mathrm{~K}$. $[\mathrm{AA}]=4.00 \times 10^{-2} \mathrm{~mol} \mathrm{dm}^{-3} ;[\mathrm{CAN}]=4.00 \times 10^{-2} \mathrm{~mol} \mathrm{dm}{ }^{-3}$; [PEG-300] $=3.33 \times 10^{-1} \mathrm{~mol} \mathrm{dm}^{-3}$.

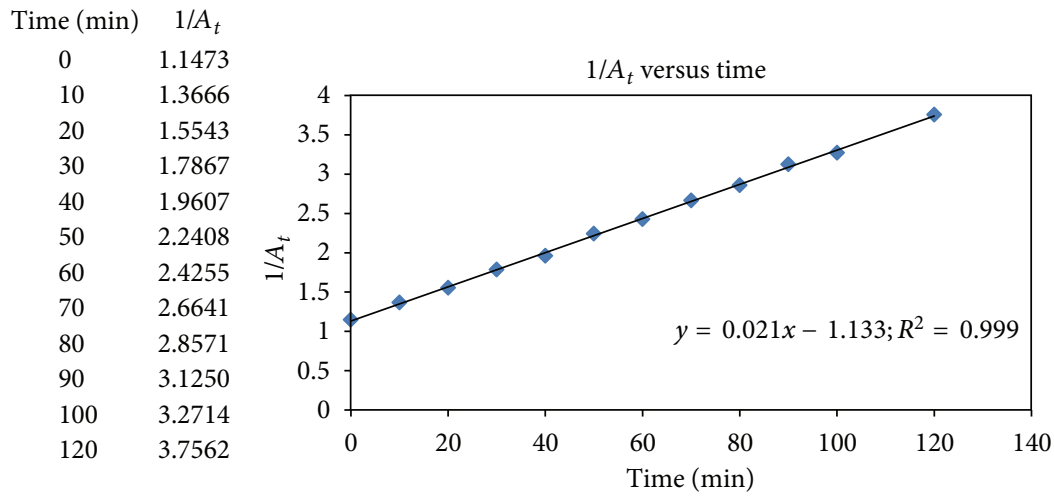

FIGURE 8: Second order kinetic plot of nitrocinnamic acid $\left(1 / A_{t}\right.$ versus time $)$ at $310 \mathrm{~K}$. $[\mathrm{NCA}]=4.00 \times 10^{-2} \mathrm{~mol} \mathrm{dm}^{-3}$; $[\mathrm{CAN}]=4.00 \times$ $10^{-2} \mathrm{~mol} \mathrm{dm}^{-3}$; [PEG-600] $=3.32 \times 10^{-1} \mathrm{~mol} \mathrm{dm}^{-3}$.

Substitution of [CAN] in (15) gives

$$
\begin{aligned}
C_{S} & =\frac{[\mathrm{CAN}-\mathrm{PEG}]}{K[\mathrm{PEG}]}+[\mathrm{CAN}-\mathrm{PEG}] \\
& =\frac{[\mathrm{CAN}-\mathrm{PEG}]+K[\mathrm{PEG}][\mathrm{CAN}-\mathrm{PEG}]}{K[\mathrm{PEG}]} \text { or } \\
& {[\mathrm{CAN}-\mathrm{PEG}]=\frac{K[\mathrm{PEG}] C_{\mathrm{S}}}{1+K[\mathrm{PEG}]} . }
\end{aligned}
$$

Similarly free substrate $[\mathrm{CAN}]$ is written as $[\mathrm{CAN}]=C_{S}-$ [CAN-PEG]

$$
[\mathrm{CAN}]=C_{S}-\frac{K[\mathrm{PEG}] C_{S}}{1+K[\mathrm{PEG}]} .
$$

After simplification, the previous equation reduces to

$$
[\mathrm{CAN}]=\frac{C_{S}}{1+K[\mathrm{PEG}]}
$$

Substitution of [CAN-PEG] and [CAN] in (15) gives

$$
\begin{gathered}
k^{\prime}=\frac{k_{0} C_{S}}{1+K[\mathrm{PEG}]}+\frac{k_{m} K[\mathrm{PEG}] C_{S}}{1+K[\mathrm{PEG}]} \text { or } \\
k_{\varphi}=\frac{\left(k_{0}+k_{m} K[\mathrm{PEG}]\right)}{1+K[\mathrm{PEG}]} \\
k_{\varphi}=\frac{\left(k_{0}+k_{m} K[\mathrm{PEG}]\right)}{1+K[\mathrm{PEG}]},
\end{gathered}
$$

where $k_{\varphi}=\left(k^{\prime} /[\mathrm{CAN}]\right)$, the second order rate constant in PEG media. Subtracting $k_{0}$ from both sides of equation and rearranging

$$
k_{\varphi}-k_{0}=\frac{\left(k_{m}-k_{w}\right) K[\mathrm{PEG}]}{1+K[\mathrm{PEG}]} .
$$

But, since the reactions are too sluggish in the absence of [PEG], the rate constant $\left(k_{0}\right)$ would be much smaller than $\left(k_{m} K[\mathrm{PEG}]\right)$; that is, $\left(k_{0} \lll k_{m} K[\mathrm{PEG}]\right)$. Therefore the $\left(k_{0}\right)$ term could be neglected in the previous equation. The rate law (21) could be then considered as

$$
k_{\varphi}=\frac{k_{m} K[\mathrm{PEG}]}{1+K[\mathrm{PEG}]} .
$$




\begin{tabular}{|c|l|}
\hline $10^{3} / T$ & $\ln \left(\mathrm{k}^{\prime \prime} / T\right)$ \\
\hline 3.3333 & -8.9226 \\
\hline 3.2786 & -8.4334 \\
\hline 3.225 & -7.9491 \\
\hline 3.1746 & -7.562 \\
\hline
\end{tabular}

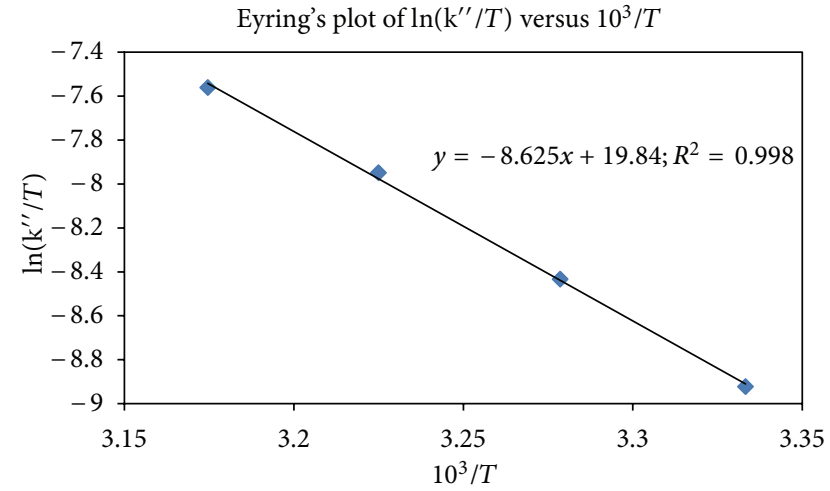

FIgURE 9: Eyring's plot of cinnamic acid $\left(\ln \left(k^{\prime \prime} / T\right)\right.$ versus $\left.10^{3} / T\right)$. Kinetic study with [PEG-200] $=2.00 \mathrm{~mol} \mathrm{dm}^{-3}$.

\begin{tabular}{|l|l|}
\hline $10^{3} / T$ & $\ln \left(k^{\prime \prime} / T\right)$ \\
\hline 3.3333 & -10.3089 \\
\hline 3.2786 & -9.6323 \\
\hline 3.225 & -9.0431 \\
\hline 3.1746 & -8.4118 \\
\hline
\end{tabular}

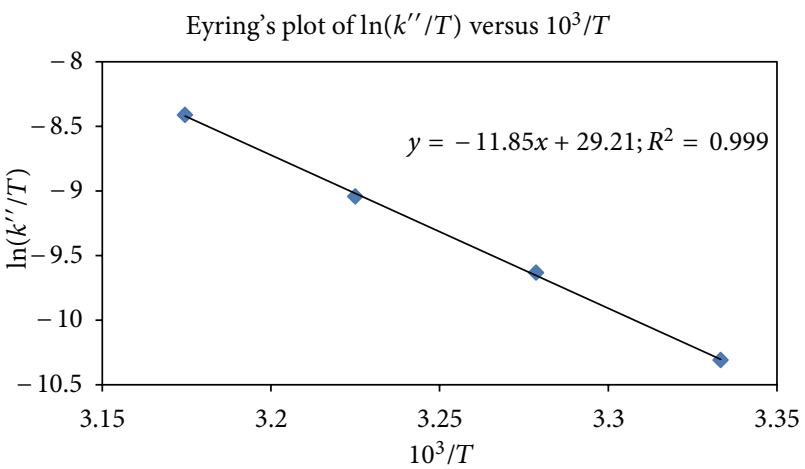

FIGURE 10: Eyring's plot of acrylic acid $\left(\ln \left(k^{\prime \prime} / T\right)\right.$ versus $\left.10^{3} / T\right)$. Kinetic study [PEG-300] $=13.32 \times 10^{-1} \mathrm{~mol} \mathrm{dm}^{-3}$.

This rate-law resembles Michaelis-Menten type rate law that is used for enzyme kinetics. Interestingly the plots of rate constant $\left(k_{\varphi}\right)$, that is, second order rate constant of PEG-mediated reaction versus [PEG], indicated either Hill type curve (i.e., a gradual increase with an increase in [PEG] passing through a maximum point in the profile). This observation could be explained due to the CAN-PEG binding interactions to give [CAN-PEG] species which is responsible for rate accelerations in PEG-mediated reactions. Formation of [CAN-PEG] species has also been established by UV-Visible spectroscopic studies, and binding constants are compiled in Table 1. These observations strengthened us to take up detailed reaction kinetics at various PEG concentrations in order to have an insight into the variation in the enthalpies and entropies of activation with [PEG].

3.4. Effect of Structure on the Reactivity, Enthalpy, and Entropy Changes. The enthalpy $\left(\Delta H^{\#}\right)$ and entropy of activation $\left(\Delta S^{\#}\right)$ are the two parameters typically obtained from the temperature dependence of a reaction rate, when these data are analyzed using Eyring's equation. Both of these values are obtained from Eyring's plots according to standard procedures [37-39]. Typical plots are shown in Figures 9 and 10 . The entropy of activation $\left(\Delta S^{\#}\right)$ provides clues about the molecularity of the rate determining step in a reaction, that is, whether the reactants are bonded to each other or not. Even though structural change in the cinnamic acid exhibits slight variation on its reactivity, no quantitative conclusions could be obtained from rate constant data. However, the observed positive $\Delta S^{\#}$ values in lower PEG concentration (0.1 M PEG) decreased with an increase in [PEG] and are becoming more negative for all the unsaturated acids which probably indicates that PEG is tightly bound to CAN and lends support that PEG bound CAN is the active species in PEG-mediated reactions. The observed negative $\Delta S^{\#}$ values are in consonance with the concept that for associative mechanism, entropy decreases upon achieving the transition state, and values near zero are difficult to interpret [38-40]. By and large similar magnitude of observed free energy of activation $\left(\Delta G^{\#}\right)$ in this reaction series (Tables $2,3,4,5,6$ ) indicates a similar type of mechanism which is operative in the present study. The observed negative magnitude of $\Delta G$ (Table 1) indicates the spontaneity of complexation of [CANPEG] due to PEG binding with CAN.

\section{Conclusions}

Being a versatile chemical reagent CAN has been applied to organic reactions in catalytic or stoichiometric amounts. Our research group has succeeded in using CAN to perform 
nitrodecarboxylation of $\alpha, \beta$-unsaturated aromatic carboxylic acids to give $\beta$-nitrostyrene derivatives. Furthermore, use of PEG supported CAN could effectively initiate the decarboxylation of carboxyl group followed by nitration. Because the CAN reagent is cost effective, we believe that the present protocol offers a rapid and clean alternative and reduces reaction times.

\section{References}

[1] T. L. Ho, "Ceric ion oxidation in organic chemistry," Synthesis, vol. 6, pp. 347-354, 1973.

[2] V. Nair, L. Balagopal, R. Rajan, and J. Mathew, "Recent advances in synthetic transformations mediated by cerium(IV) ammonium nitrate," Accounts of Chemical Research, vol. 37, no. 1, pp. 21-30, 2004.

[3] J. R. Hwu and K. Y. King, "Versatile reagent ceric ammonium nitrate in modern chemical synthesis," Current Science, vol. 81, no. 8, pp. 1043-1053, 2001.

[4] V. Nair and A. Deepthi, "Cerium(IV) ammonium nitrate-a versatile single-electron oxidant," Chemical Reviews, vol. 107, no. 5, pp. 1862-1891, 2007.

[5] V. Singh, V. Sapehiyia, and G. L. Kad, "Ultrasonically activated oxidation of hydroquinones to quinones catalysed by ceric ammonium nitrate doped on metal exchanged K-10 clay," Synthesis, no. 2, pp. 198-200, 2003.

[6] H. M. Chawla and R. S. Mittal, "Oxidative nitration by silica gelsupported cerium(IV) ammonium nitrate," Synthesis, vol. 1, pp. 70-72, 1985.

[7] S. Dincturk and J. H. J. Ridd, "Nitration of an electron-rich aromatic compound by $\mathrm{CAN}-\mathrm{SiO}_{2}$," Journal of the Chemical Society, Perkin Transactions, vol. 2, pp. 965-969, 1982.

[8] A. S. Sarac, "Redox polymerization," Progress in Polymer Science, vol. 24, no. 8, pp. 1149-1204, 1999.

[9] I. E. Marko, A. Ates, A. Gautier et al., "Cerium(IV)-catalyzed deprotection of acetals and ketals under mildly basic conditions," Angewandte Chemie International Edition, vol. 38, no. 21, pp. 3207-3209, 1999.

[10] N. Maulide, J. C. Vanherck, A. Gautier, and I. E. Markó, "Mild and neutral deprotections catalyzed by cerium(IV) ammonium nitrate," Accounts of Chemical Research, vol. 40, no. 6, pp. 381392, 2007.

[11] J. Christoffers, T. Werner, and M. Rössle, "Cerium-catalyzed oxidative $\mathrm{C}-\mathrm{C}$ bond forming reactions," Catalysis Today, vol. 121, no. 1-2, pp. 22-26, 2007.

[12] M. Rössle, T. Werner, W. Frey, and J. Christoffers, "Ceriumcatalyzed, aerobic oxidative synthesis of 1,2-dioxane derivatives from styrene and their fragmentation into 1,4-dicarbonyl compounds," European Journal of Organic Chemistry, no. 23, pp. 5031-5038, 2005.

[13] E. Ganin and I. Amer, "Cerium-catalyzed selective oxidation of alkylbenzenes with bromate salts," Synthetic Communications, vol. 25, no. 20, pp. 3149-3154, 1995.

[14] K. Auty, B. C. Gilbert, C. B. Thomas, S. W. Brown, C. W. Jones, and W. R. Sanderson, "The selective oxidation of toluenes to benzaldehydes by cerium(III), hydrogen peroxide and bromide ion," Journal of Molecular Catalysis A, vol. 117, no. 1-3, pp. 279287, 1997.

[15] R. P. Kreh, R. M. Spotnitz, and J. T. Lundquist, "Mediated electrochemical synthesis of aromatic aldehydes, ketones, and quinones using ceric methanesulfonate," Journal of Organic Chemistry, vol. 54, no. 7, pp. 1526-1531, 1989.

[16] H. Feuer and A. T. Nielsen, Nitro Compounds Recent Advances in Synthesis and Chemistry, vol. 9, VCH Publishers, New York, NY, USA, 1990.

[17] A. G. M. Barrett, "Heterosubstituted nitroalkenes in synthesis," Chemical Society Reviews, vol. 20, no. 1, pp. 95-127, 1991.

[18] R. S. Varma and G. W. Kabalka, "Nitroalkenes in the synthesis of heterocyclic compounds," Heterocycles, vol. 24, no. 9, pp. 26452677, 1986.

[19] S. E. Denmark and L. R. Marcin, "A general method for the preparation of 2,2-disubstituted 1-nitroalkenes," Journal of Organic Chemistry, vol. 58, no. 15, pp. 3850-3856, 1993.

[20] M. Node, A. Itoh, K. Nishide et al., "Preparation of nitroalkenes: Substitution reaction via addition-elimination using $\beta$ nitrovinyl sulfoxides," Synthesis, no. 11, pp. 1119-1124, 1992.

[21] R. Ballini, R. Castagnani, and M. Petrini, "Chemoselective synthesis of functionalized conjugated nitroalkenes," Journal of Organic Chemistry, vol. 57, no. 7, pp. 2160-2162, 1992.

[22] S. S. Jew, H. D. Kim, Y. S. Cho, and C. H. Cook, "A practical preparations of conjugated nitroalkenes," Chemistry Letters, vol. 1986, no. 10, pp. 1747-1748.

[23] W. W. Sy and A. W. By, "Nitration of substituted styrenes with nitryl iodide," Tetrahedron Letters, vol. 26, no. 9, pp. 1193-1196, 1985.

[24] V. V. Namboodiri and R. S. Varma, "Microwave-accelerated Suzuki cross-coupling reaction in polyethylene glycol (PEG)," Green Chemistry, vol. 3, no. 3, pp. 146-148, 2001.

[25] A. Haimov and R. Neumann, "Polyethylene glycol as a nonionic liquid solvent for polyoxometalate catalyzed aerobic oxidation," Chemical Communications, no. 8, pp. 876-877, 2002.

[26] L. Heiss, "Polyethylene glycol monomethyl ether-modified pig liver esterase: Preparation, characterization and catalysis of enantioselective hydrolysis in water and acylation in organic solvents," Tetrahedron Letters, vol. 36, no. 22, pp. 3833-3836, 1995.

[27] S. Chandrasekhar, C. Narsihmulu, S. S. Sultana, and N. R. Reddy, "Osmium tetroxide in poly(ethylene glycol) (PEG): a recyclable reaction medium for rapid asymmetric dihydroxylation under sharpless conditions," Chemical Communications, vol. 9, no. 14, pp. 1716-1717, 2003.

[28] K. Tanemura, T. Suzuki, Y. Nishida, and T. Horaguchi, "Aldol condensation in water using polyethylene glycol 400," Chemistry Letters, vol. 34, no. 4, pp. 576-577, 2005.

[29] R. Kumar, P. Chaudhary, S. Nimesh, and R. Chandra, "Polyethylene glycol as a non-ionic liquid solvent for Michael addition reaction of amines to conjugated alkenes," Green Chemistry, vol. 8, no. 4 , pp. $356-358,2006$.

[30] J. R. Johnson, “The perkin reaction and related reactions," Organic Reactions, vol. 1, p. 210, 1942.

[31] C. I. Chiriac, F. Tanasa, and M. Onciu, "A novel approach in cinnamic acid synthesis: direct synthesis of cinnamic acids from aromatic aldehydes and aliphatic carboxylic acids in the presence of boron tribromide," Molecules, vol. 10, no. 2, pp. 481487, 2005.

[32] H. A. Benesi and J. H. Hildebrand, "Ultraviolet absorption bands of iodine and aromatic hydrocarbons," Journal of the American Chemical Society, vol. 71, pp. 1832-1833, 1949.

[33] M. Santappa and B. Sethuram, "Oxidation studies. IV. Kinetics of oxidation of $\mathrm{HCHO}$ and some alcohols by ceric salts in $\mathrm{HNO}_{3}$ medium," Proceedings of the Indian Academy of Sciences Section $A$, vol. 67 , no. 2, pp. 78-89, 1968. 
[34] K. V. Rao and S. S. Muhammed, "Oxidation of alcohols by cerium(IV). I. Oxidation of methanol by ceric perchlorate," Bulletin of the Chemical Society of Japan, vol. 36, no. 8, pp. 943949, 1963.

[35] S. S. Muhammed and B. Sethuram, "Oxidation of alcohols by cerium(IV). I. Oxidation of isopropanol and secondary butanol by ceric nitrate," Acta Chimica Hungarica, vol. 46, pp. 115-124, 1965.

[36] N. Dutt, R. R. Nagori, and R. N. Mehrotra, "Kinetics and mechanisms of oxidations by metal ions. Part VI. Oxidation of $\alpha$-hydroxyl acids by cerium (IV) in aqueous nitric acid," Canadian Journal of Chemistry, vol. 64, no. 1, pp. 19-23, 1986.

[37] F. M. Menger and C. E. Portnoy, "Chemistry of reactions proceeding inside molecular aggregates," Journal of the American Chemical Society, vol. 89, no. 18, pp. 4698-4730, 1967.

[38] K. A. Connors, Chemical Kinetics: The Study of Reaction Rates in Solution, VCH, New York, NY, USA, 1990.

[39] J. H. Espenson, Chemical Kinetics and Reaction Mechanism, McGraw-Hill, New York, NY, USA, 1981.

[40] V. Jagannadham, "The change in entropy of activation due to solvation (hydration) of ions: proton versus carbocation," Chemistry (the Bulgarian Journal of Chemical Education), vol. 18 , p. $89,2009$. 

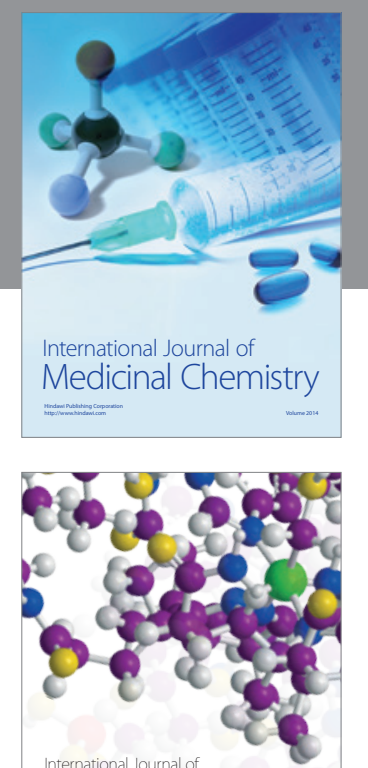

\section{Carbohydrate} Chemistry

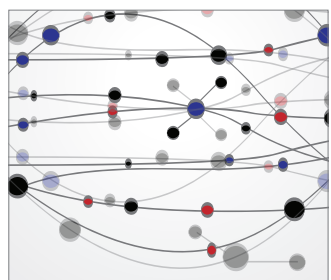

The Scientific World Journal
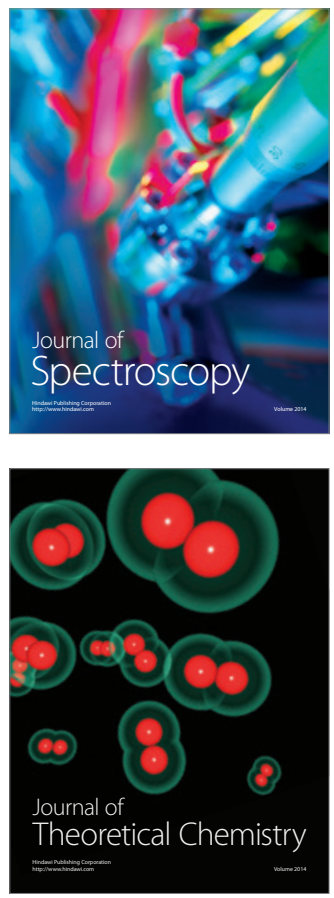
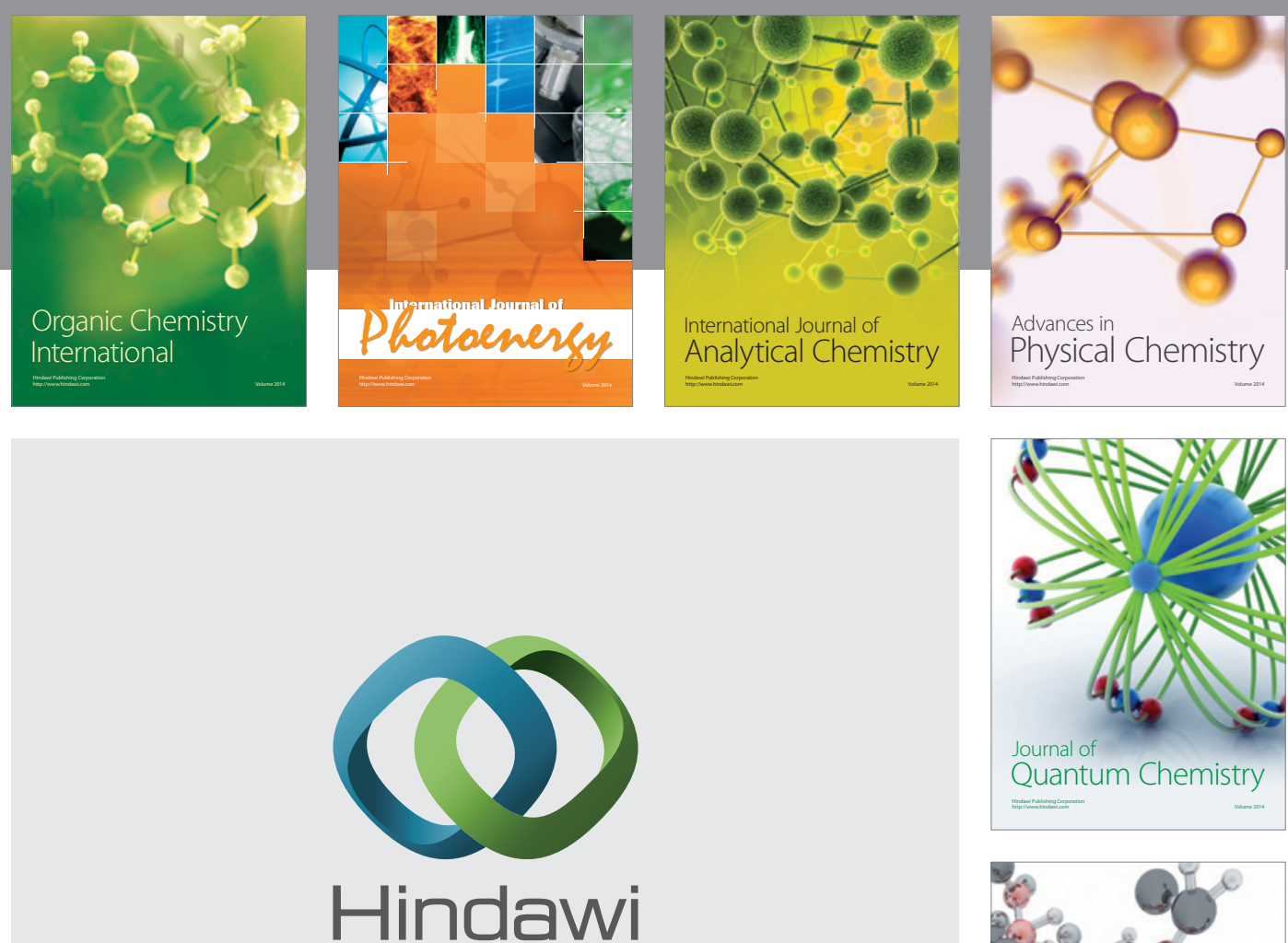

Submit your manuscripts at

http://www.hindawi.com

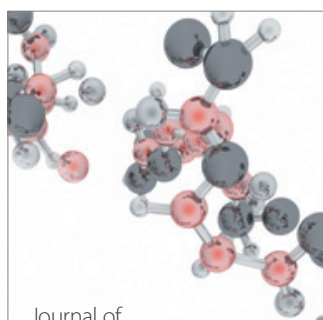

Analytical Methods

in Chemistry

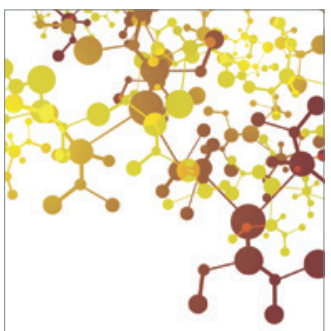

Journal of

Applied Chemistry

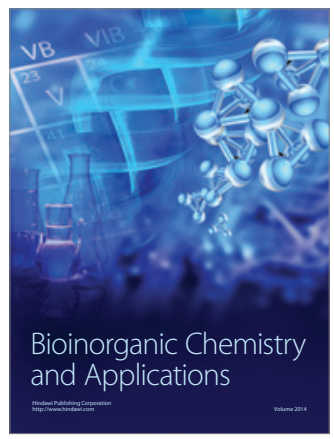

Inorganic Chemistry
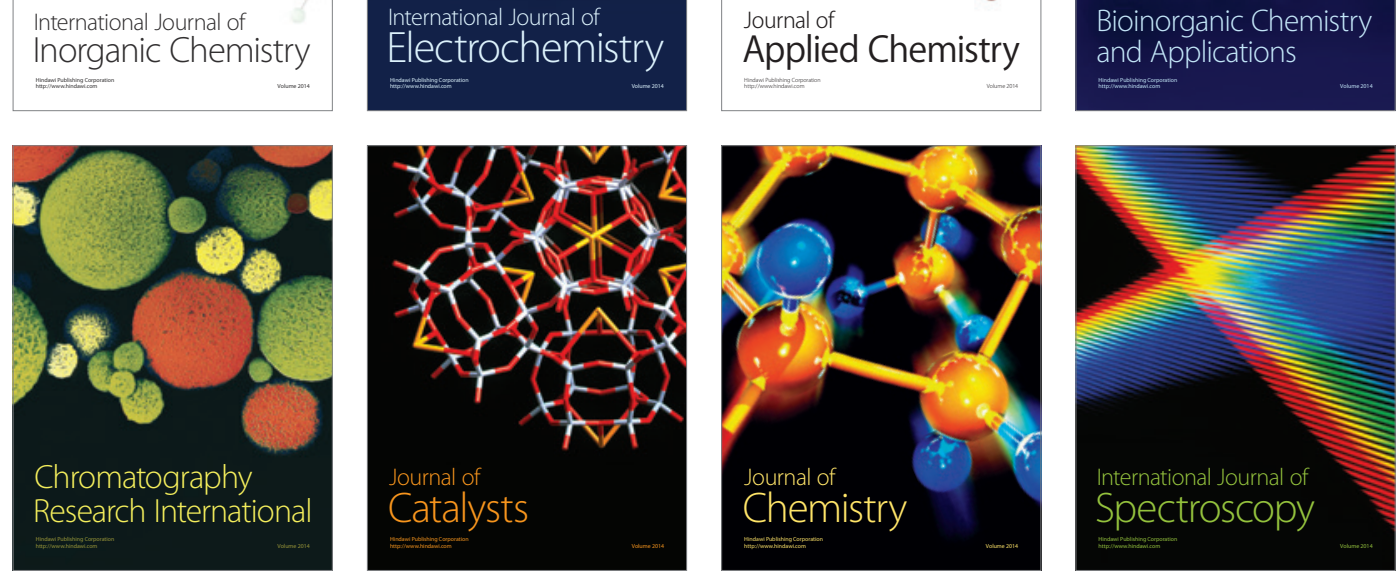